\title{
Quality control for the first large areas of triple-GEM chambers for the CMS endcaps
}

D. Abbaneo ${ }^{17}$, M. Abbas ${ }^{17}$, M. Abbrescia ${ }^{2}$, M. Abi Akl ${ }^{13}$, O. Aboamer ${ }^{8}$, D. Acosta ${ }^{15}$, A. Ahmad ${ }^{19}$, W. Ahmed ${ }^{19}$, A. Aleksandrov ${ }^{28}$, P. Altieri ${ }^{2}$, C. Asawatangtrakuldee ${ }^{3}$, P. Aspell ${ }^{17}$, Y. Assran ${ }^{8}$, I. Awan ${ }^{19}$, S. Bally ${ }^{17}$, Y. Ban ${ }^{3}$, S. Banerjee ${ }^{20}$, V. Barashko ${ }^{15}$, P. Barria ${ }^{5}$, G. Bencze ${ }^{7}$, N. Beni ${ }^{10}$, L. Benussi ${ }^{14}$, V. Bhopatkar ${ }^{23}$, S. Bianco ${ }^{14}$, J. Bos $^{17}$, O. Bouhali ${ }^{13}$, A. Braghieri' ${ }^{26}$, S. Braibant ${ }^{4}$, S. Buontempo ${ }^{25}$, C. Calabria ${ }^{2}$, M. Caponero ${ }^{14}$, C. Caputo ${ }^{2}$, F. Cassese ${ }^{25}$, A. Castaneda ${ }^{13}$, S. Cauwenbergh ${ }^{18}$, F.R. Cavallo ${ }^{4}$, A. Celik ${ }^{9}$, M. Choi ${ }^{32}$, S. Choi ${ }^{30}$, J. Christiansen ${ }^{17}$, A. Cimmino ${ }^{18}$, S. Colafranceschi ${ }^{17}$, A. Colaleo ${ }^{2}$, A. Conde Garcia ${ }^{17}$, S. Czellar ${ }^{10}$, M.M. Dabrowski ${ }^{17}$, G. De Lentdecker $^{5}$, R. De Oliveira ${ }^{17}$, G. de Robertis ${ }^{2}$, S. Dildick ${ }^{9,18}$, B. Dorney ${ }^{17}$, G. Endroczi ${ }^{7}$, F. Errico ${ }^{2}$, A. Fenyvesi ${ }^{10}$, S. Ferry ${ }^{17}$, I. Furic ${ }^{15}$, P. Giacomelli ${ }^{4}$, J. Gilmore ${ }^{9}$, V. Golovtsov ${ }^{16}$, L. Guiducci ${ }^{4}$, F. Guilloux ${ }^{27}$, A. Gutierrez ${ }^{12}$, R.M. Hadjiiska ${ }^{28}$, J. Hauser ${ }^{22}$, K. Hoepfner ${ }^{1}$, M. Hohlmann ${ }^{23}$, H. Hoorani ${ }^{19}$, P. laydjiev ${ }^{28}$, Y.G. Jeng ${ }^{32}$, T. Kamon ${ }^{9}$, P. Karchin ${ }^{12}$, A. Korytov ${ }^{15}$, S. Krutelyov ${ }^{9}$, A. Kumar ${ }^{11}$, H. Kim ${ }^{32}$, J. Lee ${ }^{32}$, T. Lenzi ${ }^{5}$, L. Litov ${ }^{29}$, F. Loddo ${ }^{2}$, A. Madorsky ${ }^{15}$, T. Maerschalk ${ }^{5}$, M. Maggi ${ }^{2}$, A. Magnani ${ }^{26}$, P.K. Mal ${ }^{6}$, K. Mandal ${ }^{6}$, A. Marchioro ${ }^{17}$, A. Marinov ${ }^{17}$, N. Majumdar ${ }^{20}$, J.A. Merlin ${ }^{17,33}$, G. Mitselmakher ${ }^{15}$, A.K. Mohanty ${ }^{24}$, A. Mohapatra ${ }^{23}$, J. Molnar ${ }^{10}$, S. Muhammad ${ }^{19}$, S. Mukhopadhyay ${ }^{20}$, M. Naimuddin ${ }^{11}$, S. Nuzzo ${ }^{2}$, E. Oliveri ${ }^{17}$, L.M. Pant ${ }^{24}$, P. Paolucci ${ }^{25}$, I. Park ${ }^{32}$, G. Passeggio ${ }^{25}$, B. Pavlov ${ }^{29}$, B. Philipps ${ }^{1}$, D. Piccolo ${ }^{14}$, H. Postema ${ }^{17}$, A. Puig Baranac ${ }^{17}$, A. Radi ${ }^{8}$, R. Radogna ${ }^{2}$, G. Raffone ${ }^{14}$, A. Ranieri' ${ }^{2}$, G. Rashevski ${ }^{28}$, C. Riccardi ${ }^{26}$, M. Rodozov ${ }^{28}$, A. Rodrigues ${ }^{17}$, L. Ropelewski ${ }^{17}$, S. RoyChowdhury ${ }^{20}$, G. Ryu ${ }^{32}$, M.S. Ryu ${ }^{32}$, A. Safonov ${ }^{9}$, S. Salva ${ }^{18}$, G. Saviano ${ }^{14}$, A. Sharma ${ }^{2}$, A. Sharma ${ }^{17}$, R. Sharma ${ }^{11}$, A.H. Shah ${ }^{11}$, M. Shopova ${ }^{28}$, J. Sturdy ${ }^{12}$, G. Sultanov ${ }^{28}$, S.K. Swain ${ }^{6}$, Z. Szillasi ${ }^{10}$, J. Talvitie ${ }^{21}$, A. Tatarinov ${ }^{9}$, T. Tuuva ${ }^{21}$, M. Tytgat ${ }^{18, a}$, I. Vai ${ }^{26}$, M. Van Stenis ${ }^{17}$, R. Venditti ${ }^{2}$, E. Verhagen ${ }^{5}$, P. Verwilligen ${ }^{2}$, P. Vitulo ${ }^{26}$, S. Volkov ${ }^{16}$, A. Vorobyev ${ }^{16}$, D. Wang ${ }^{3}$, M. Wang ${ }^{3}$, U. Yang ${ }^{31}$, Y. Yang ${ }^{5}$, R. Yonamine ${ }^{5}$, N. Zaganidis ${ }^{18}$, F. Zenoni ${ }^{5}$, and A. Zhang ${ }^{23}$

${ }^{1}$ RWTH Aachen University, III Physikalisches Institut A, Aachen, Germany

${ }^{2}$ INFN Bari and University of Bari, Bari, Italy

${ }^{3}$ Peking University, Beijing, China

${ }^{4}$ INFN Bologna and University of Bologna, Bologna, Italy

${ }^{5}$ Universite Libre de Bruxelles, Brussels, Belgium

${ }^{6}$ National Institute of Science Education and Research, Bhubaneswar

${ }^{7}$ Institute for Particle and Nuclear Physics, Wigner Research Centre for Physics, Hungarian Academy of Sciences, Budapest, Hungary

${ }^{8}$ Academy of Scientific Research and Technology - Egyptian Network of High Energy Physics, ASRT-ENHEP, Cairo, Egypt

${ }^{9}$ Texas A\&M University, College Station, U.S.A.

${ }^{10}$ Institute for Nuclear Research of the Hungarian Academy of Sciences (ATOMKI), Debrecen, Hungary

${ }^{11}$ University of Delhi, Delhi, India

${ }^{12}$ Wayne State University, Detroit, U.S.A

${ }^{13}$ Texas A\&M University at Qatar, Doha, Qatar

${ }^{14}$ Laboratori Nazionali di Frascati - INFN, Frascati, Italy

${ }^{15}$ University of Florida, Gainesville, U.S.A.

${ }^{16}$ Petersburg Nuclear Physics Institute, Gatchina, Russia

${ }^{17}$ CERN, Geneva, Switzerland

${ }^{18}$ Ghent University, Dept. of Physics and Astronomy, Ghent, Belgium

${ }^{19}$ National Center for Physics, Quaid-i-Azam University Campus, Islamabad, Pakistan

${ }^{20}$ Saha Institute of Nuclear Physics, Kolkata, India

${ }^{21}$ Lappeenranta University of Technology, Lappeenranta, Finland

${ }^{22}$ University of California, Los Angeles, U.S.A.

${ }^{23}$ Florida Institute of Technology, Melbourne, U.S.A.

${ }^{24}$ Bhabha Atomic Research Centre, Mumbai, India

${ }^{25}$ INFN Napoli, Napoli, Italy

${ }^{26}$ INFN Pavia and University of Pavia, Pavia, Italy

${ }^{27}$ IRFU CEA-Saclay, Saclay, France

${ }^{28}$ Institute for Nuclear Research and Nuclear Energy, Sofia, Bulgaria

${ }^{29}$ Sofia University, Sofia, Bulgaria

${ }^{30}$ Korea University, Seoul, Korea 


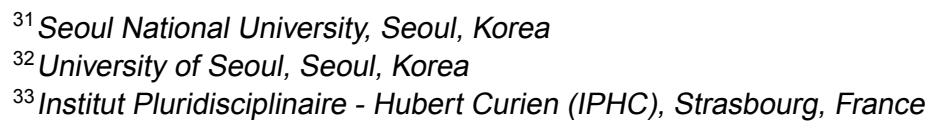

\begin{abstract}
The CMS Collaboration plans to equip the very forward muon system with triple-GEM detectors that can withstand the environment of the High-Luminosity LHC. This project is at the final stages of R\&D and moving to production. An unprecedented large area of several $100 \mathrm{~m}^{2}$ are to be instrumented with GEM detectors which will be produced in six different sites around the world. A common construction and quality control procedure is required to ensure the performance of each detector. The quality control steps will include optical inspection, cleaning and baking of all materials and parts used to build the detector, leakage current tests of the GEM foils, high voltage tests, gas leak tests of the chambers and monitoring pressure drop vs. time, gain calibration to know the optimal operation region of the detector, gain uniformity tests, and studying the efficiency, noise and tracking performance of the detectors in a cosmic stand using scintillators.
\end{abstract}

\section{Introduction}

The CMS Collaboration is planning several muon system upgrades in order to maintain its high level of performance achieved during LHC Run 1 also during the future High Luminosity LHC [1]. A quadrant of the CMS muon system is shown in Figure 1, where both the present detectors and the proposed extensions are displayed. In the $1.5<|\eta|<2.4$ forward endcap region, currently only Cathode Strip Chambers are installed. To increase redundancy and enhance the muon trigger and reconstruction capabilities in that region, additional muon detectors are foreseen. In particular, two new stations, called GE1/1 and GE2/1, would be equipped with GEM detectors. Furthermore, in order to increase the coverage for muon detection up to $|\eta| \approx 3$, another GEM station called ME0 is proposed to be installed behind new endcap calorimeters.

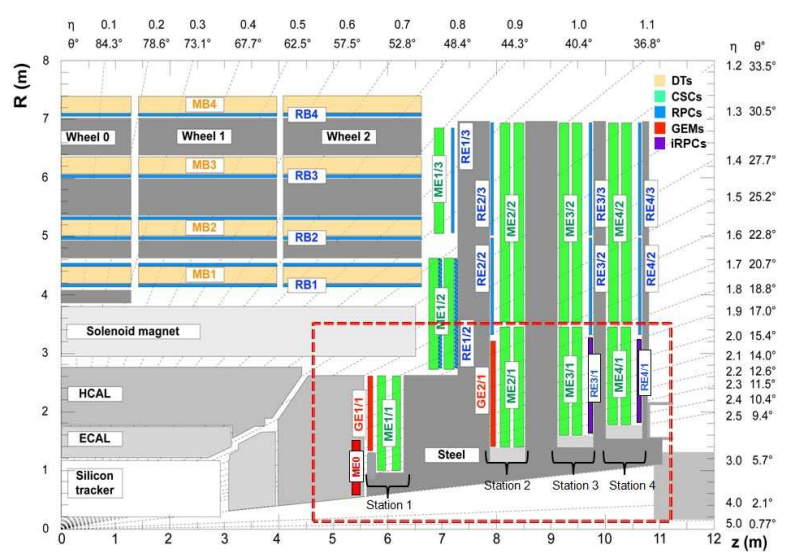

Figure 1. Quadrant of the CMS muon system showing present detectors, i.e. Drift Tubes (DTs), Resistive Plate Chambers (RPCs) and Cathode Strip Chambers (CSCs), and the locations of the proposed GEM detectors, i.e. the ME0, GE1/1 and GE2/1 stations.

Preparations for a demonstrator consisting of four GE1/1 superchambers covering a $40^{\circ}$ sector are well advanced; their installation is foreseen during the LHC 2016 Year End Technical Stop. The full GE1/1 station is scheduled for installation in CMS during LHC Long Shutdown

\footnotetext{
${ }^{\mathrm{a} C}$ Corresponding author; e-mail: Michael.Tytgat@UGent.be
}

2 (2018-2020). The installation of GE2/1 and ME0 chambers is planned for Long Shutdown 3 (2024-2026).

\section{The GE1/1 Station}

For the muon detectors to be installed in the CMS $1.5<$ $|\eta|<2.2$ endcap region in the face of the high luminosity, GEMs are an excellent choice due to their thin profile and the ability to operate well at the high particle fluxes expected there under HL-LHC conditions. The GE1/1 station will be equipped with triple-GEM chambers that were developed over the years starting in 2009 [2]. The detector front-end electronics will be based on the custom designed VFAT3 chip, providing both fast input to the CMS level-1 muon trigger and full granularity information for the offline muon reconstruction. A full description of the GE1/1 station can be found in [3].

\subsection{Chambers and Superchambers}

The GE1/1 stations to be installed in each of the two CMS endcaps consist of 36 superchambers, each made of a double layer of trapezoidal triple-GEM chambers that cover about $10^{\circ}$ in $\phi$. The superchambers alternate in $\phi$ between long $(1.5<|\eta|<2.18)$ and short $(1.6<|\eta|<2.18)$ versions to maximize the coverage of the available space. The total thickness of a superchamber is $88 \mathrm{~mm}$.

The 144 GE1/1 chambers needed to produce the 72 superchambers in total, have a trapezoidal shape with a $10.15^{\circ}$ opening angle and are $3.5 \mathrm{~cm}$ thick. They contain a triple-GEM detector with a $3 / 1 / 2 / 1 \mathrm{~mm}$ drift/transfer$1 /$ transfer-2/induction field gap configuration. The active readout area is $0.345 / 0.409 \mathrm{~m}^{2}$ for the short/long chambers. The foils for the short/long chambers are segmented in $40 / 47 \mathrm{HV}$ sectors and in $3 \times 8$ readout sectors in $\phi \times \eta$, each with 128 readout strips, yielding a total of 3072 readout channels per chamber. The chambers will be operated with an $\mathrm{Ar} / \mathrm{CO}_{2}$ 70:30 gas mixture, at a nominal detector gain of $1-2 \times 10^{4}$.

\subsection{Detector Production and Quality Control}

The GEM foils are produced at the CERN Surface Treatment Workshop using a single-mask technique. A new 


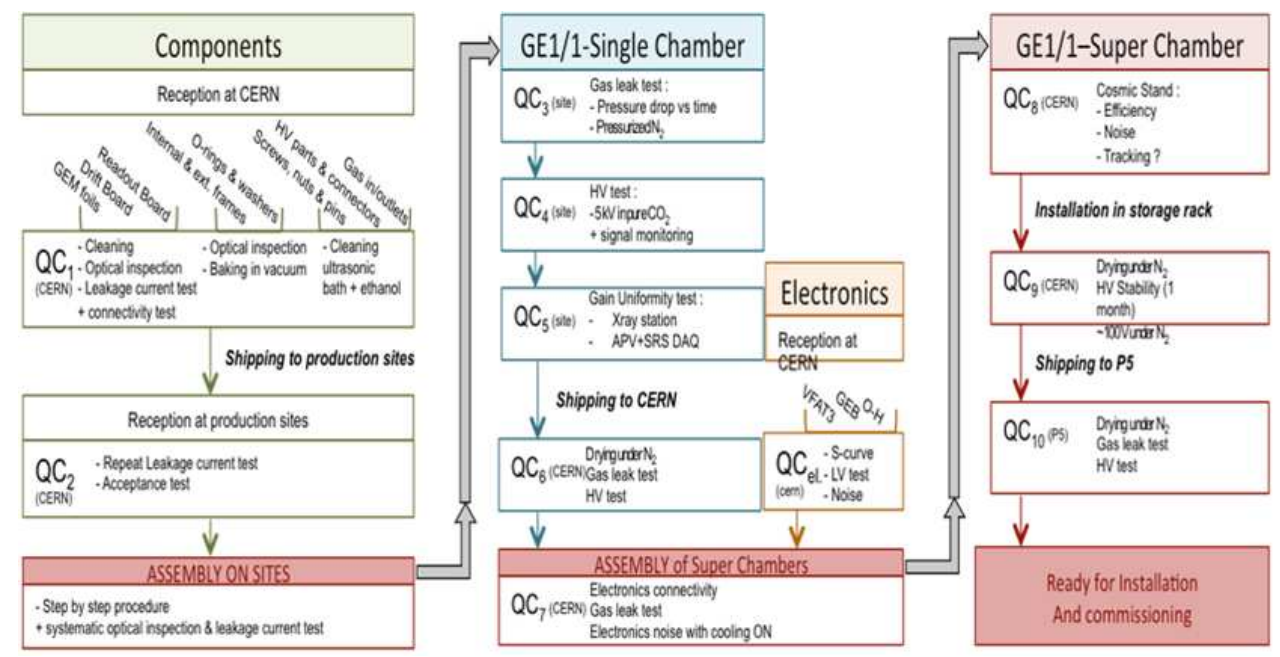

Figure 2. GE1/1 chamber and superchamber assembly and quality control workflow.

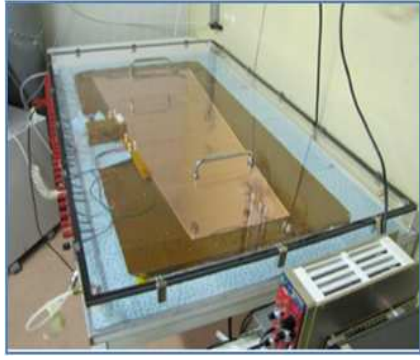

Figure 3. Leakage current measurement station for the GE1/1 GEM foils.

technique in which the three GEM foils of a chamber are simultaneously stretched in a mechanical way, allows to assemble a full chamber in a few hours time [4].

As depicted in Figure 2, the general GE1/1 chamber and superchamber assembly and quality control workflow consists of 3 main parts:

- Component production and quality control (QC1-2)

- Assembly and commissioning of GE1/1 chambers at production sites (QC3-5)

- Assembly and commissioning of superchambers at CERN before installation in CMS (QC6-10)

After cleaning of all parts using ultrasonic baths, baking and sand blast procedures, the inspection of the main chamber components includes an I-V curve measurement of the voltage divider, an optical and electrical inspection of the drift board, and a verification of the readout board checking for possible shorts between readout strips. The GEM foils are optically inspected for defects and then submitted to a leakage current test (see Figure 3) in which the foils should not draw a current higher than 30nA when a potential difference of $500 \mathrm{~V}$ is applied between the GEM metal sides.

Upon completion of the assembly, every chamber is checked for gas leaks by pressurizing it up to $20 \mathrm{mbar}$

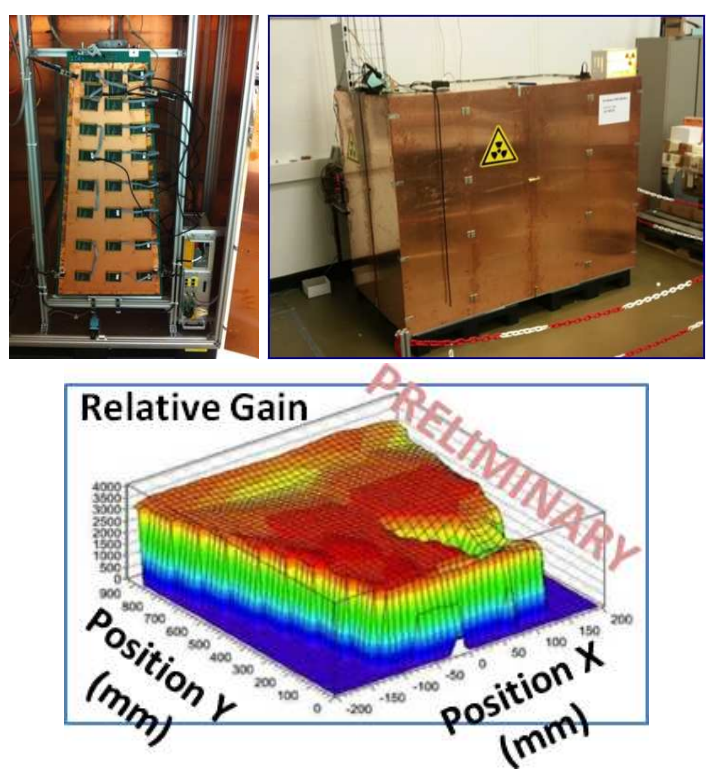

Figure 4. The chamber gain uniformity measurements, including pictures of the setup at CERN (top) and an example graph showing the relative gain of the chamber as function the position along its surface.

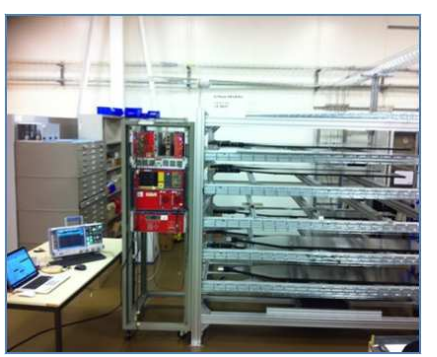

Figure 5. Cosmic stand at CERN for the final quality control of GE1/1 superchambers. 

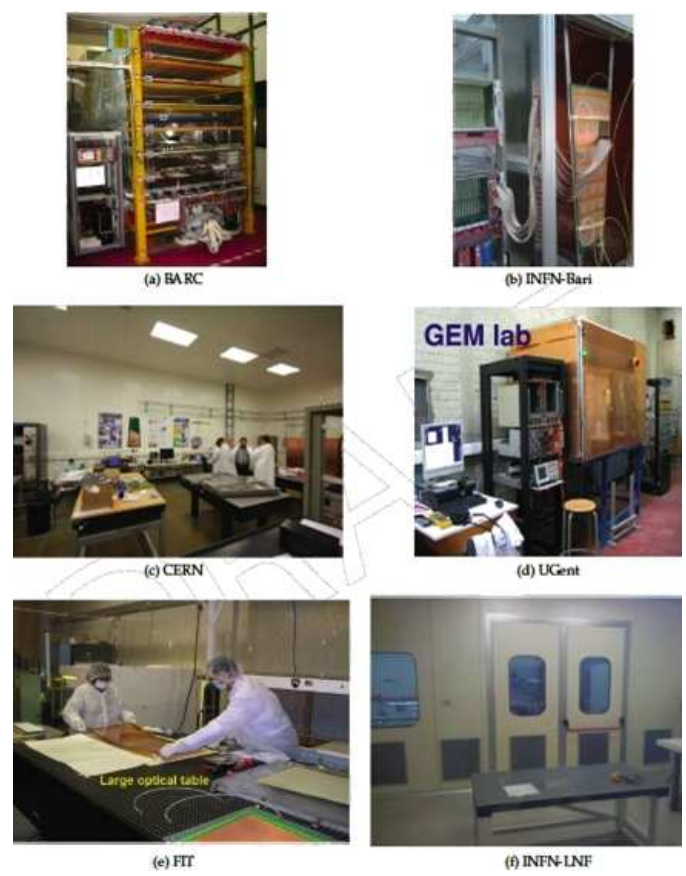

Figure 6. The following assembly sites for GE1/1 production are being considered by CMS: (a) Bhabha Atomic Research Center (BARC), India; (b) INFN Sezione di Bari, Italy; (c) CERN, Switzerland; (d) Ghent University, Belgium; (e) Florida Institute of Technology (FIT), USA; (f) INFN Laboratori Nazionali di Frascati (LNF), Italy.

with dry nitrogen. Next, the output signal of the chambers is monitored after flushing for several hours with $\mathrm{Ar} / \mathrm{CO}_{2}$ and turning them on at moderate HV. Furthermore, the uniformity of the gain of each chamber across its surface is verified in a dedicated setup inside a Copper protective box (see Figure 4), with an SRS/APV25 hybrid based dataacquisition [5] and an X-ray source placed at a distance of about $1 \mathrm{~m}$, illuminating the full chamber at once.

Superchambers are fabricated by coupling together two GE1/1 single chambers. Inside a cosmic stand, a high voltage scan is performed on each chamber and the relevant parameters such as detector gain, noise levels and cluster size are measured with the final detector electronics. Figure 5 shows the present cosmic stand at CERN, which allows to test up to 15 superchambers at once and features fully automated HV scans, measurements of cosmic muon tracks and a DAQ system comparable to the final setup to be used inside the CMS experiment.

Throughout the entire detector assembly and quality control procedure, all detailed information and test results will be stored in an Oracle based database for future reference.

\subsection{Assembly Sites}

At present, six assembly sites are being considered for GE1/1 mass production. All sites will follow the same assembly and quality control protocols and will include at least the following setups:

- A certified cleanroom of class 1000 minimum to assemble full GE1/1 chambers; the cleanroom must be equipped with clean and dry nitrogen gas lines used to blow chamber parts during the assembly

- A nitrogen-flushed box to measure GEM foil leakage currents at voltages up to $500 \mathrm{~V}$

- An X-ray setup for gain uniformity measurements across the chamber surface

- A gas leak measurement station for verify gas tightness of the chambers

Pictures of different assembly site candidates are shown in Figure 6.

\section{Summary}

Facing the High-Luminosity LHC, the CMS Collaboration is planning several muon system upgrades in order to maintain its high level performance in terms of muon triggering and reconstruction. CMS recently approved the installation of the so-called GE1/1 station: during LHC Long Shutdown 2, the $1.5<|\eta|<2.2$ region of the first endcap disks will be equipped with a total of 144 new triple-GEM detectors, arranged in 72 super-chambers. The GE1/1 chamber assembly will be done at several locations in- and outside CERN; a detailed chamber assembly and quality control workflow is being worked out.

\section{Acknowledgements}

We gratefully acknowledge support from FRS-FNRS (Belgium), FWO-Flanders (Belgium), BSF-MES (Bulgaria), BMBF (Germany), DAE (India), DST (India), INFN (Italy), NRF (Korea), QNRF (Qatar), and DOE (USA).

\section{References}

[1] The CMS Collaboration, Technical Proposal for the Phase-II Upgrade of the CMS Detector, CERN-LHCC2015-010, LHCC-P-008

[2] D. Abbaneo et al., JINST 9 (2014) 10, C10036

[3] The CMS Collaboration, CMS Technical Design Report for the Muon Endcap GEM Upgrade, CERNLHCC-2015-012, CMS-TDR-013

[4] D. Abbaneo et al., Nucl. Instrum. Meth. A732, 203207 (2013)

[5] S. Martoiu et al., 2011 IEEE Nucl. Sci. Symp. Conf. Rec., 2036-2038 (2011) 